\title{
Aprendizaje colectivo: la experiencia "Asociativismo" de la Universidad Nacional de General Sarmiento
}

\section{Cecilia Chosco Díaz y Viviana Ramallo}

Universidad Nacional de General Sarmiento, Argentina.

\section{Resumen}

En Argentina, durante estos últimos años, se desarrollaron experiencias universitarias orientadas al fortalecimiento de las organizaciones sociales. En esta dirección, "Asociativismo" de la Universidad Nacional de General Sarmiento (desarrollada entre los años 2013 y 2015), representa una forma de aprender haciendo, y de crear nuevo conocimiento, para el análisis organizacional, entre docentes universitarios y organizaciones sociales. En tal sentido, se evidencia una forma de aprendizaje colectivo, siguiendo la estrategia de los enfoques del aprendizaje y el servicio solidario; y el enfoque interdisciplinario. En efecto, esto permitió comprender avances, fortalezas, oportunidades, debilidades y amenazas de las asociaciones civiles, las fundaciones y las cooperativas.

\section{Palabras clave}

Aprendizaje colectivo, organizaciones sociales, diagnóstico organizacional.

Fecha de recepción: 20/VIII/2014

Fecha de aceptación: 14/V/2015 


\title{
Collective learning: The experience "Asociativismo" of the Universidad Nacional de General Sarmiento
}

\begin{abstract}
In Argentina, during these last years, college experiences aimed to the strengthening social organizations were developed. In this direction, "Asociativismo" de la Universidad Nacional de General Sarmiento (developed between 2013 and 2015), represents a form of learn doing with the other, and create new knowledge, to the organizational analysis, between university professors and social organizations. In this regard, it is evident a form of collective learning, following the strategy of the learning approaches and the solidary service; and the interdisciplinary approach. In effect, this allowed understand progress, strengths, weaknesses, opportunities y threats of the civil associations, foundations and cooperatives, from the reports and the experiences of their referents.
\end{abstract}

\section{Keywords}

Collective learning, social organizations, organizational diagnosis. 


\section{Introducción}

¿Qué es "Asociativismo"? Es un servicio con la comunidad conformado por un equipo interdisciplinario de profesionales de Administración Pública, Política Social, Educación, Ingeniería y Economía Industrial. Actualmente participa un estudiante de Economía Industrial y Ecología. Se inicia en el año 2013 a través de un subsidio de la Secretaría de Políticas Universitarias y continúa entre el 2014 y 2015, mediante el octavo Fondo Estímulo de la Universidad Nacional de General Sarmiento.

¿Por qué "Asociativismo"? Surge como una necesidad de los graduados y profesionales para fortalecer las capacidades de las organizaciones sociales y con el compromiso de fortalecer las redes en el territorio. Las actividades (encuentros debate) siguieron el enfoque basado en el aprendizaje y el servicio solidario (Tapia, 2008). Es una propuesta compleja e innovadora que aporta la novedad de vincular estrechamente el servicio y el aprendizaje en una sola actividad educativa articulada y coherente. La misma representa una forma de aprender haciendo con el otro. Es también una innovación pedagógica en la que la solidaridad se convierte en una forma de aprender, en un modo de dar sentido y relevancia social al conocimiento. No es beneficencia. Por el contrario, propone un modelo de solidaridad comprometida y responsable frente a las necesidades del otro.

\section{Antecedentes: el aprendizaje servicio solidario}

El enfoque aprendizaje servicio solidario, a nivel internacional, tiene 
sus orígenes en los años sesenta. Ha sido estudiado ampliamente por el campo de la educación y de la psicología. Es reconocida por los aportes de Robert Sigmon (1979), Timothy Stanton (1987), Honnet y Poulsen (1989), como así también por diversos investigadores de la National Society for Experiential Education y de la Service Learning in Education Reform y por la Red Iberoamericana de Aprendizaje Servicio (CLAYSS). A nivel nacional, se implementa en los años noventa, con la reforma de la educación nacional a partir de la Ley 26206 y los programas pertinentes. En la actualidad la Red Universitaria REXUNI, desarrolla talleres y capacitaciones con este enfoque.

¿Qué entendemos por el aprendizajeservicio solidario? es una forma de aprendizaje activo y significativo, situado en el contexto de una comunidad que se convierte en lugar de participación y también en lugar de aprendizaje (Tapia, 2008). La autora sostiene que es posible generar una intersección entre dos tipos de experiencias educativas que generalmente se desarrollan aisladamente. Por un lado, actividades en función de un aprendizaje interdisciplinario y por otro, se desarrollan actividades solidarias de una causa de la propia comunidad u organización. Por lo tanto, entendemos que existe un aprendizaje servicio, cuando se genera la intersección entre la intencionalidad pedagógica y solidaria. Este enfoque permitió reconstruir historias, vivencias y realizar análisis sobre las asociaciones civiles, fundaciones y cooperativas a través de una praxis interdisciplinaria, la cual es entendida como aquella 
práctica cuyo propósito es la transformación de la realidad natural, social y de los sujetos, y la permanente generación de múltiples formas de conocimientos. De este modo, se fue consolidando otra pedagogía de análisis organizacional. Ahora bien para el desarrollo de la misma es fundamental la interdisciplinariedad. Según Japiassu (1976) la interdisciplinariedad constituye un principio nuevo de reorganización de las disciplinas científicas y una reformulación de estructuras pedagógicas de enseñanza. Se busca producir un discurso para problemas concretos. Se confrontan e interactúan puntos de vista de varias disciplinas, donde el objetivo no es crear una nueva disciplina científica ni tampoco un discurso universal sino resolver lo concreto. En esas condiciones, las prácticas interdisciplinares pueden ser consideradas como negociaciones entre puntos de vista, entre proyectos e intereses diferentes. Parafraseando al autor, es una práctica política que supone una interpenetración o interfecundación, desde las más simples comunicaciones e ideas hasta la integración mutua de conceptos (contratos interdisciplinares), de la epistemología y de la metodología, de los procedimientos, de los datos y de la organización de investigaciones.

En efecto, la complementariedad de los enfoques redistribuye las relaciones de poder-saber, destierra las prácticas asistencialistas y promueve el fortalecimiento del capital social entre la universidad y las organizaciones sociales. 




\section{La experiencia: el caso de}

\section{"Asociativismo"}

En esta experiencia se siguieron las etapas del método interdisciplinario (Japiassu, 1976), específicamente cuatro: la creación de un equipo de trabajo, la unificación de lenguaje, el estudio y análisis de problemas organizacionales y la puesta en práctica de espacios de aprendizaje colectivo. Durante la primera etapa se constituyó un equipo de trabajo, allí se consensuó y reflexionó entre el grupo de profesionales la posibilidad emprender un proyecto colectivo, en el que cada docente fuese capaz de exponer sus competencias, contribuciones y limitaciones de su disciplina en relación a las organizaciones sociales. Para el abordaje organizacional, se contó con la participación de diversos profesionales de Administración,
Administración Pública, Educación, Política Social, Comunicación, y un estudiante de grado de la carrera en Economía Industrial y Ecología. Asimismo, se concibió a las organizaciones sociales como sistemas abiertos, complejos y dinámicos (Etkin, 2007) tendientes a la autoorganización y a la creación de un conocimiento propio. En la segunda etapa, se tendió a unificar el lenguaje técnico como así también a planificar una forma específica para interactuar con las organizaciones participantes. En este sentido, se delinearon estrategias pedagógicas que favorecieran el intercambio de opiniones, de diálogos, críticas, a través de la reflexión colectiva. Así fueron emergiendo ejes de análisis de las organizaciones tal como la estructura, el planeamiento, las redes, las decisiones estratégicas y 
diversas capacidades institucionales. Paulatinamente se fue generando una praxis entre diversos saberes que tendieron a ser un conocimiento colectivo y progresivo. Actualmente estos encuentros se denominan "Escuchando a las organizaciones" y ya se realizaron cinco jornadas de las cuales participaron diversas entidades: Cooperativistas del Programa "Ellas Hacen", Cooperativa Alcoyanna, Cooperativa Madygraf (ExDonellys), Cooperativa de Trabajo Curtidores de Hurlingham Ltda., Cooperativa 103 de José C Paz, Asociación Ambientalista Bonaerense (ASAMBO), SUTEBA SAN MIGUEL, CTA SAN MIGUEL, Cámara Empresarial e Industrial de Malvinas Argentinas, Unión Empresarial de Moreno, Observatorio Permanente de Organizaciones Sociales de la Universidad Nacional de Luján,
Asociación Argentina de Formación Profesional, Fundación OfICIOS, Centro de Formación y Promoción de Derechos Primavera, Brigada Ecológica Juvenil de San Miguel, Unión de Familias Obreras, Cámara de Comercio de Don Torcuato, Servicio Paz y Justicia (SERPAJ), Fundación de la Universidad Nacional de General Sarmiento, R.S.T MACACHA GÜEMES, Asociación Civil Pequeños Pasos, Asamblea del Delta y Río de la Plata, Asociación civil Albert Einsteinn y la Escuela Regional de Reforestación, entre otras.

\section{Resultados}

La experiencia promovió el afianzamiento de los vínculos con y entre diversos actores locales y regionales. Asimismo generó procesos de doble transferencia de conocimiento y circularidad de la 
información entre docentes, estudiantes, y los referentes de las organizaciones. Posibilitó la emergencia de nuevas formas de conocimiento mediante el aprendizaje colectivo, teniendo en cuenta la perspectiva territorial, social, y crítica, basada en experiencias y vivencias de referentes, y de los docentes. También, hacia adentro de la universidad fomentó la creación de equipos de jóvenes docentes investigadores orientados a la implementación de servicios con la comunidad.

\section{Referencias bibliográficas}

Etkin, J. (2007). La capacidad de gobernar en organizaciones complejas. Los acuerdos, la tensión creativa y tolerancia a la diversidad. Cali: Universidad del Valle.

Tapia, L. (2008) Política Salvaje. La Paz: CLACSO. 